\title{
On the norms of $r$-circulant matrices with generalized Fibonacci numbers
}

\section{Amara Chandoul}

Abstract: In this paper, we obtain a generalization of [6, 8]. Firstly, we consider the so-called $r$-circulant matrices with generalized Fibonacci numbers and then found lower and upper bounds for the Euclidean and spectral norms of these matrices. Afterwards, we present some bounds for the spectral norms of Hadamard and Kronecker product of these matrices.

2010 MSC: 15B05, 15A60, 65F35

Keywords: Matrix norm, $r$-circulant matrices, Generalized Fibonacci numbers

\section{Introduction}

The Fibonacci numbers are the elements of integer sequence $\left\{F_{n}\right\}_{n=0}^{\infty}$ defined by the linear recurrence equation

$$
\left\{\begin{array}{l}
F_{0}=0, F_{1}=1 \\
F_{n+2}=F_{n+1}+F_{n}, \text { for } n=0,1,2, \ldots
\end{array}\right.
$$

The sequence is s given by

$$
0,1,1,2,3,5,8,13, \ldots
$$

You cannot go very far in the lore of Fibonacci numbers without encountering the companion sequence of Lucas numbers $\left\{L_{n}\right\}_{n=0}^{\infty}$, which follows the same recursive pattern as the Fibonacci numbers, but begins with other initial values. It is defined by the linear recurrence equation

$$
\left\{\begin{array}{l}
L_{0}=2, L_{1}=1 \\
L_{n+2}=L_{n+1}+L_{n}, \text { for } n=0,1,2, \ldots
\end{array}\right.
$$

Amara Chandoul; Departamento de Matemática, Universidade de Brasília, Campus Universitário Darcy Ribeiro Brasília - DF 70910-900, Brazil (email: amarachandoul@yahoo.fr). 
The sequence is s given by

$$
2,1,3,4,7,11,18,29, \ldots
$$

The Fibonacci sequence has been studied extensively and generalized in many ways. Such generalizations are possible in four directions, namely, by changing the initial values, by mixing two Lucas sequences, by not demanding that the numbers in the sequences be integers, or by having more than two parameters.

One of generalization of Fibonacci i sequence is the integer sequence $\left\{G_{n}\right\}_{n=0}^{\infty}$. It has the same recurrence relation as Fibonacci and Lucas, namely it is defined by the linear recurrence equation

$$
\left\{\begin{array}{l}
G_{0}=a, G_{1}=b \\
G_{n+2}=G_{n+1}+G_{n}, \text { for } n=0,1,2, \ldots
\end{array}\right.
$$

The starting values of $G_{0}=a$ and $G_{1}=b$ can be specified. It therefore includes both sequences them both as special cases.

This generalized Fibonacci sequence is giving by

$$
a, b,(a+b),(a+2 b),(2 a+3 b),(3 a+5 b),(5 a+8 b),(8 a+13 b), \ldots
$$

$\left\{G_{n}\right\}_{n=0}^{\infty}$ is a generalization of $\left\{F_{n}\right\}_{n=0}^{\infty}$ and $\left\{L_{n}\right\}_{n=0}^{\infty}$. Furthermore, there is a relationship such that $G_{n}=a F_{n-1}+b F_{n}, a, b \in \mathbb{R}$ between Fibonacci and generalized Fibonacci numbers.

Fibonacci, Lucas numbers and their generalization have many intersting properties and applications to almost every field of science and art. For the beauty and rich applications of these numbers and their relatives one may see Vajda's and Koshy's books [7, 12].

Solak [10] defined the circulant matrices with Fibonacci and Lucas numbers, and he obtained lower and upper bounds with the Fibonacci number for the Euclidean and spectral norms of these matrices.

Civciv and Türkmen [2] constructed the circulant matrix with the Lucas number and then presented lower and upper bounds for the Euclidean and spectral norms of this matrix as a function of $n$ and $L_{n}$ which is $n$th Lucas number. In [2], they are studied the norm bounds for the Hadamard inverse of this matrix. In [1], authors computed some norms of r-circulant matrices associated with a number sequence.

In this study, we first construct the so-called $r$-circulant matrix with the generalized Fibonacci numbers and then present some lower and upper bounds for the Euclidean and spectral norms of this matrix.

We begin with some preliminaries related to our study. A matrix $C=\left[c_{i j}\right] \in \mathcal{M}_{n}(\mathbb{C})$ is called a $r$-circulant matrix if it is of the form

$$
c_{i j}= \begin{cases}c_{j-i} & j \geq i \\ r c_{n+j-i} & j<i .\end{cases}
$$

Obviously, the $r$-circulant matrix $C$ is determined by parameter $r$ and its first row elements $c_{0}, c_{1}, \cdots, c_{n-1}$. Especially, for $r=1$, the matrix $C$ is called a circulant matrix. For any $A=\left[a_{i j}\right] \in$ $\mathcal{M}_{m, n}(\mathbb{C})$, the well known Frobenius (or Euclidean) norm of matrix $A$ is

$$
\|A\|_{E}=\sqrt{\sum_{i=1}^{n} \sum_{j=1}^{n}\left|a_{i j}\right|^{2}}
$$

The spectral norm of the matrix $A$

$$
\|A\|_{2}=\sqrt{\max _{1 \leq i \leq n} \lambda_{i}\left(A^{H} A\right)}
$$


where $\lambda_{i}$ is an eigenvalue of $A^{H} A$ and $A^{H}$ is conjugate transpose of the matrix $A$. It is well known that

$$
\frac{1}{\sqrt{n}}\|A\|_{E} \leq\|A\|_{2} \leq\|A\|_{E} .
$$

If $\|$.$\| is any norm on m \times n$ complex matrices, then [5]

$$
\|A \circ B\| \leq\|A\|\|B\|,
$$

where $A \circ B$ is the Hadamard product of $A$ and $B$. Define the maximum column lenght norm $c_{1}(A)$ and the maximum row lenght norm $r_{1}(A)$ of any matrix A respectively by

$$
c_{1}(A)=\max _{1 \leq j \leq n} \sqrt{\sum_{i=1}^{n}\left|a_{i j}\right|^{2}}
$$

and

$$
r_{1}(A)=\max _{1 \leq i \leq n} \sqrt{\sum_{j=1}^{n}\left|a_{i j}\right|^{2}} .
$$

Let $A, B$ and $C$ be $m \times n$ complex matrices. if $A=B \circ C$, then [4]

$$
\|A\|_{2} \leq r_{1}(B) c_{1}(C)
$$

\section{Results}

Definition 2.1. The r-circulant matrix A with generalized Fibonacci number is the matrix of the form

$$
A=\left(\begin{array}{ccccc}
G_{0} & G_{1} & \ldots & \ldots & G_{n-1} \\
r G_{n-1} & G_{0} & \ldots & \ldots & G_{n-2} \\
r G_{n-2} & r G_{n-1} & \ddots & \ldots & G_{n-3} \\
\vdots & \vdots & \ddots & G_{0} & \vdots \\
r G_{1} & r G_{2} & \ldots & r G_{n-1} & G_{0}
\end{array}\right)
$$

Theorem 2.2. Let $A$ be the matrix defined in (3). Then

(i) If $|r| \geq 1$, then

$$
\|A\|_{E} \geq \sqrt{n\left(G_{n-1} G_{n}-G_{1} G_{0}+G_{0}^{2}\right)},\|A\|_{2} \geq \sqrt{G_{n-1} G_{n}-G_{1} G_{0}+G_{0}^{2}}
$$

and

$$
\|A\|_{2} \leq \min \left\{\begin{array}{l}
\sqrt{\left((n-1)|r|^{2}+1\right)\left(G_{n-1} G_{n}-G_{1} G_{0}+G_{0}^{2}\right)}, \\
\sqrt{\left((n-1)|r|^{2}+G_{0}^{2}\right)\left(G_{n-1} G_{n}-G_{1} G_{0}+1\right)}, \\
\sqrt{\left(G_{n-1} G_{n}-G_{1} G_{0}+G_{0}^{2}\right)\left(G_{n-1} G_{n}-G_{1} G_{0}+1\right)}
\end{array}\right.
$$

Moreover, if $G_{0}=0$, then

$$
\sqrt{G_{n} G_{n-1}} \leq\|A\|_{2} \leq|r| G_{n-1} G_{n}
$$


(ii) If $|r|<1$, then

$$
\|A\|_{E} \geq|r| \sqrt{n\left(G_{n-1} G_{n}-G_{1} G_{0}+G_{0}^{2}\right)},\|A\|_{2} \geq|r| \sqrt{G_{n-1} G_{n}-G_{1} G_{0}+G_{0}^{2}}
$$

and

$$
\|A\|_{2} \leq \min \left\{\begin{array}{l}
\sqrt{n\left(G_{n-1} G_{n}-G_{1} G_{0}+G_{0}^{2}\right)}, \\
\sqrt{\left((n-1)+G_{0}^{2}\right)\left(G_{n-1} G_{n}-G_{1} G_{0}+1\right)}, \\
\sqrt{\left((n-1)+G_{0}^{2}\right)\left(G_{n-1} G_{n}-G_{1} G_{0}+G_{0}^{2}\right)} .
\end{array}\right.
$$

Moreover, if $G_{0}=0$, then

$$
|r| \sqrt{G_{n} G_{n-1}} \leq\|A\|_{2} \leq \sqrt{(n-1) G_{n-1} G_{n}}
$$

Proof. From (3), we have

$$
\|A\|_{E}=\sqrt{\sum_{k=0}^{n-1}(n-k) G_{k}^{2}+\sum_{k=1}^{n-1} k|r|^{2} G_{k}^{2}}
$$

Let matrices be defined as

$$
\begin{aligned}
& B_{1}=\left(b_{1, i j}\right)=\left\{\begin{array}{l}
b_{1, i j}=r, \quad i>j \\
b_{1, i j}=1, \quad i \leq j
\end{array}\right. \\
& B_{2}=\left(b_{2, i j}\right)= \begin{cases}b_{2, i j}=G_{(\bmod (j-i, n))}, & i \geq j \\
b_{2, i j}=1, & i<j\end{cases} \\
& B_{3}=\left(b_{3, i j}\right)= \begin{cases}b_{3, i j}=r, & i>j \\
b_{3, i j}=G_{0}, & i=j \\
b_{3, i j}=1, & i<j\end{cases} \\
& C_{1}=\left(c_{1, i j}\right)=\left(G_{(\bmod (j-i, n))}\right) . \\
& C_{2}=\left(c_{2, i j}\right)= \begin{cases}c_{2, i j}=r, & i>j \\
c_{2, i j}=1, & i=j \\
c_{2, i j}=G_{(\bmod (j-i, n))}, & i<j\end{cases}
\end{aligned}
$$

and

$$
C_{3}=\left(c_{3, i j}\right)= \begin{cases}c_{3, i j}=G_{(\bmod (j-i, n))}, & i \neq j \\ c_{3, i j}=1, & i=j\end{cases}
$$

such that $A=B_{k} \circ C_{k}, 1 \leq k \leq 3$. Then we have

$$
\begin{aligned}
& r_{1}\left(B_{1}\right)=\max _{1 \leq i \leq n} \sqrt{\sum_{j=1}^{n}\left|b_{1, i j}\right|^{2}}= \begin{cases}\sqrt{(n-1)|r|^{2}+1}, & |r| \geq 1 \\
\sqrt{n}, & |r|<1 .\end{cases} \\
& r_{1}\left(B_{2}\right)=\max _{1 \leq i \leq n} \sqrt{\sum_{j=1}^{n}\left|b_{2, i j}\right|^{2}}=\sqrt{G_{n-1} G_{n}-G_{1} G_{0}+G_{0}^{2}} . \\
& r_{1}\left(B_{3}\right)=\max _{1 \leq i \leq n} \sqrt{\sum_{j=1}^{n}\left|b_{3, i j}\right|^{2}}= \begin{cases}\sqrt{G_{0}^{2}+(n-1)|r|^{2}}, & |r| \geq 1 \\
\sqrt{G_{0}^{2}+(n-1)}, & |r|<1 .\end{cases}
\end{aligned}
$$




$$
\begin{aligned}
& c_{1}\left(C_{1}\right)=\max _{1 \leq j \leq n} \sqrt{\sum_{i=1}^{n}\left|c_{1, i j}\right|^{2}}=\sqrt{G_{n-1} G_{n}-G_{1} G_{0}+G_{0}^{2}} . \\
& c_{1}\left(C_{2}\right)=\max _{1 \leq j \leq n} \sqrt{\sum_{i=1}^{n}\left|c_{2, i j}\right|^{2}}=\sqrt{G_{n-1} G_{n}-G_{1} G_{0}+1} . \\
& c_{1}\left(C_{3}\right)=\max _{1 \leq j \leq n} \sqrt{\sum_{i=1}^{n}\left|c_{3, i j}\right|^{2}}=\sqrt{G_{n-1} G_{n}-G_{1} G_{0}+1} .
\end{aligned}
$$

If $G_{0}=0$, we consider the matrices

$$
\begin{gathered}
B_{4}=\left(b_{4, i j}\right)= \begin{cases}b_{4, i j}=r G_{(\bmod (j-i, n))}, & i \geq j \\
b_{4, i j}=1, & i<j .\end{cases} \\
C_{4}=\left(c_{4, i j}\right)= \begin{cases}c_{4, i j}=G_{(\bmod (j-i, n))}, & i \leq j \\
c_{4, i j}=1, & i>j .\end{cases}
\end{gathered}
$$

Then, we have

$$
\begin{aligned}
& r_{1}\left(B_{4}\right)=\max _{1 \leq i \leq n} \sqrt{\sum_{j=1}^{n}\left|b_{4, i j}\right|^{2}}=|r| \sqrt{G_{n-1} G_{n}}, \\
& c_{1}\left(C_{4}\right)=\max _{1 \leq j \leq n} \sqrt{\sum_{i=1}^{n}\left|c_{4, i j}\right|^{2}}=\sqrt{G_{n-1} G_{n}} .
\end{aligned}
$$

(i) If $|r| \geq 1$, then we have

$$
\|A\|_{E} \geq \sqrt{n\left(G_{n-1} G_{n}-G_{1} G_{0}+G_{0}^{2}\right)}
$$

Thus, we obtain from $\frac{1}{\sqrt{n}}\|A\|_{E} \leq\|A\|_{2}$,

$$
\|A\|_{2} \geq \sqrt{G_{n-1} G_{n}-G_{1} G_{0}+G_{0}^{2}}
$$

On the other hand, using (4), (5) and (2), we have

$$
\|A\|_{2} \leq \min \left\{r_{1}\left(B_{1}\right) c_{1}\left(C_{1}\right), r_{1}\left(B_{2}\right) c_{1}\left(C_{2}\right), r_{1}\left(B_{3}\right) c_{1}\left(C_{3}\right)\right\} .
$$

Thus, we have

$$
\|A\|_{2} \leq \min \left\{\begin{array}{l}
\sqrt{\left((n-1)|r|^{2}+1\right)\left(G_{n-1} G_{n}-G_{1} G_{0}+G_{0}^{2}\right)}, \\
\sqrt{\left((n-1)|r|^{2}+G_{0}^{2}\right)\left(G_{n-1} G_{n}-G_{1} G_{0}+1\right)}, \\
\sqrt{\left(G_{n-1} G_{n}-G_{1} G_{0}+G_{0}^{2}\right)\left(G_{n-1} G_{n}-G_{1} G_{0}+1\right)} .
\end{array}\right.
$$

Moreover, if $G_{0}=0$, using (6), then

$$
\sqrt{G_{n} G_{n-1}} \leq\|A\|_{2} \leq|r| G_{n-1} G_{n} .
$$

(ii) If $|r|<1$, then we have

$$
\|A\|_{E} \geq \sqrt{n|r|^{2}\left(G_{n-1} G_{n}-G_{1} G_{0}+G_{0}^{2}\right)} .
$$


Thus, we have from $\frac{1}{\sqrt{n}}\|A\|_{E} \leq\|A\|_{2}$,

$$
\|A\|_{2} \geq|r| \sqrt{\left(G_{n-1} G_{n}-G_{1} G_{0}+G_{0}^{2}\right)} .
$$

On the other hand, using (4), (5) and (2), we have

$$
\|A\|_{2} \leq \min \left\{r_{1}\left(B_{1}\right) c_{1}\left(C_{1}\right), r_{1}\left(B_{2}\right) c_{1}\left(C_{2}\right), r_{1}\left(B_{3}\right) c_{1}\left(C_{3}\right)\right\} .
$$

Thus, we have

$$
\|A\|_{2} \leq \min \left\{\begin{array}{l}
\sqrt{n\left(G_{n-1} G_{n}-G_{1} G_{0}+G_{0}^{2}\right)}, \\
\sqrt{\left((n-1)+G_{0}^{2}\right)\left(G_{n-1} G_{n}-G_{1} G_{0}+1\right)}, \\
\sqrt{\left((n-1)+G_{0}^{2}\right)\left(G_{n-1} G_{n}-G_{1} G_{0}+G_{0}^{2}\right)} .
\end{array}\right.
$$

Moreover, if $G_{0}=0$, then

$$
|r| \sqrt{G_{n} G_{n-1}} \leq\|A\|_{2} \leq \sqrt{(n-1) G_{n-1} G_{n}}
$$

Corollary 2.3. Let $G_{0}=a=0$ and $G_{1}=b=1$ be in the theorem. Then

(i) If $|r| \geq 1$, then $\sqrt{F_{n} F_{n-1}} \leq\|A\|_{2} \leq|r| F_{n} F_{n-1}$.

(ii) If $|r|<1$, then $|r| \sqrt{F_{n} F_{n-1}} \leq\|A\|_{2} \leq \sqrt{(n-1) F_{n} F_{n-1}}$.

Corollary 2.4. Let $G_{0}=a=2$ and $G_{1}=b=1$ be in the theorem. Then

(i) If $|r| \geq 1$, then

$$
\sqrt{L_{n} L_{n-1}+2} \leq\|A\|_{2} \leq \min \left\{\begin{array}{l}
\sqrt{\left((n-1)|r|^{2}+1\right)\left(L_{n} L_{n-1}+2\right)}, \\
\sqrt{\left((n-1)|r|^{2}+4\right)\left(L_{n} L_{n-1}-1\right)}, \\
\sqrt{\left(L_{n} L_{n-1}+2\right)\left(L_{n} L_{n-1}-1\right)} .
\end{array}\right.
$$

(ii) If $|r|<1$, then

$$
|r| \sqrt{L_{n} L_{n-1}+2} \leq\|A\|_{2} \leq \sqrt{(n+3)\left(L_{n} L_{n-1}-1\right)}
$$

Theorem 2.5. Let $A$ be the matrix defined in (3). Then

(i) If $|r| \geq 1$, then

$$
\|A\|_{2} \geq \begin{cases}\sqrt{a^{2}\left(1+F_{n-2} F_{n-1}\right)+2 a b F_{n-1}^{2}+b^{2} F_{n} F_{n-1}}, & n \text { odd } \\ \sqrt{a^{2}\left(1+F_{n-2} F_{n-1}\right)+2 a b\left(F_{n-1}^{2}-1\right)+b^{2} F_{n} F_{n-1}}, & n \text { even }\end{cases}
$$

(ii) If $|r|<1$, then

$$
\|A\|_{2} \geq \begin{cases}|r| \sqrt{a^{2}\left(1+F_{n-2} F_{n-1}\right)+2 a b F_{n-1}^{2}+b^{2} F_{n} F_{n-1}}, & n \text { odd } \\ |r| \sqrt{a^{2}\left(1+F_{n-2} F_{n-1}\right)+2 a b\left(F_{n-1}^{2}-1\right)+b^{2} F_{n} F_{n-1}}, & n \text { even }\end{cases}
$$


Proof. We have,

$$
\begin{aligned}
\sum_{k=0}^{n-1} G_{k}^{2} & =G_{n-1} G_{n}-G_{1} G_{0}+G_{0}^{2} \\
& =\sum_{k=0}^{n-1}\left(a F_{k-1}+b F_{k}\right)^{2}=a^{2} \sum_{k=0}^{n-1} F_{k-1}^{2}+2 a b \sum_{k=0}^{n-1} F_{k-1} F_{k}+b^{2} \sum_{k=0}^{n-1} F_{k}^{2} .
\end{aligned}
$$

As

$$
\sum_{k=0}^{n-1} F_{k-1}^{2}=F_{n-1} F_{n-2}+1, \sum_{k=0}^{n-1} F_{k}^{2}=F_{n} F_{n-1}
$$

and

$$
\sum_{k=0}^{n-1} F_{k-1} F_{k}= \begin{cases}F_{n-1}^{2}, & n \text { odd } \\ F_{n-1}^{2}-1, & n \text { even }\end{cases}
$$

The proof of Theorem 2.5 become trivial.

Theorem 2.6. Let $A$ be the matrix defined in (3). Then

(i) If $|r| \geq 1$, then

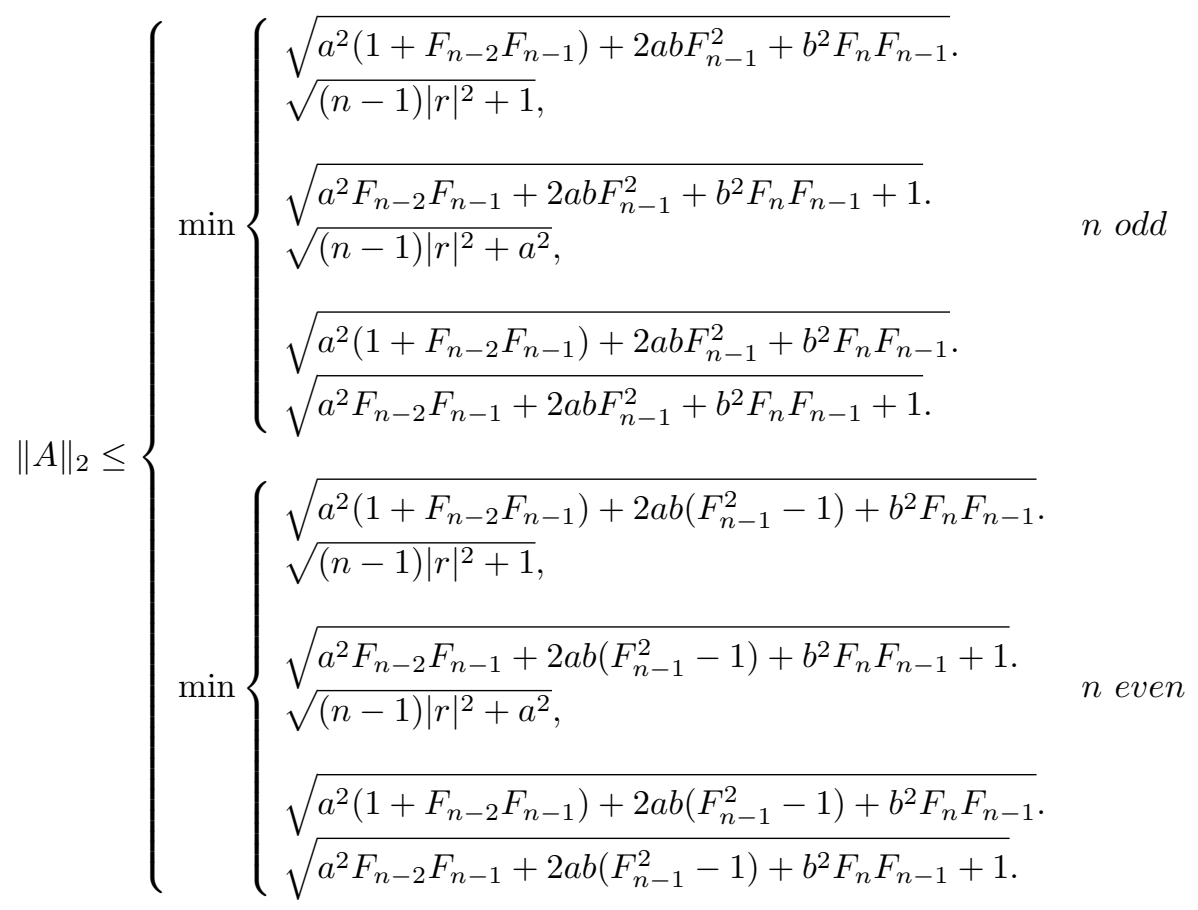


(ii) If $|r|<1$, then

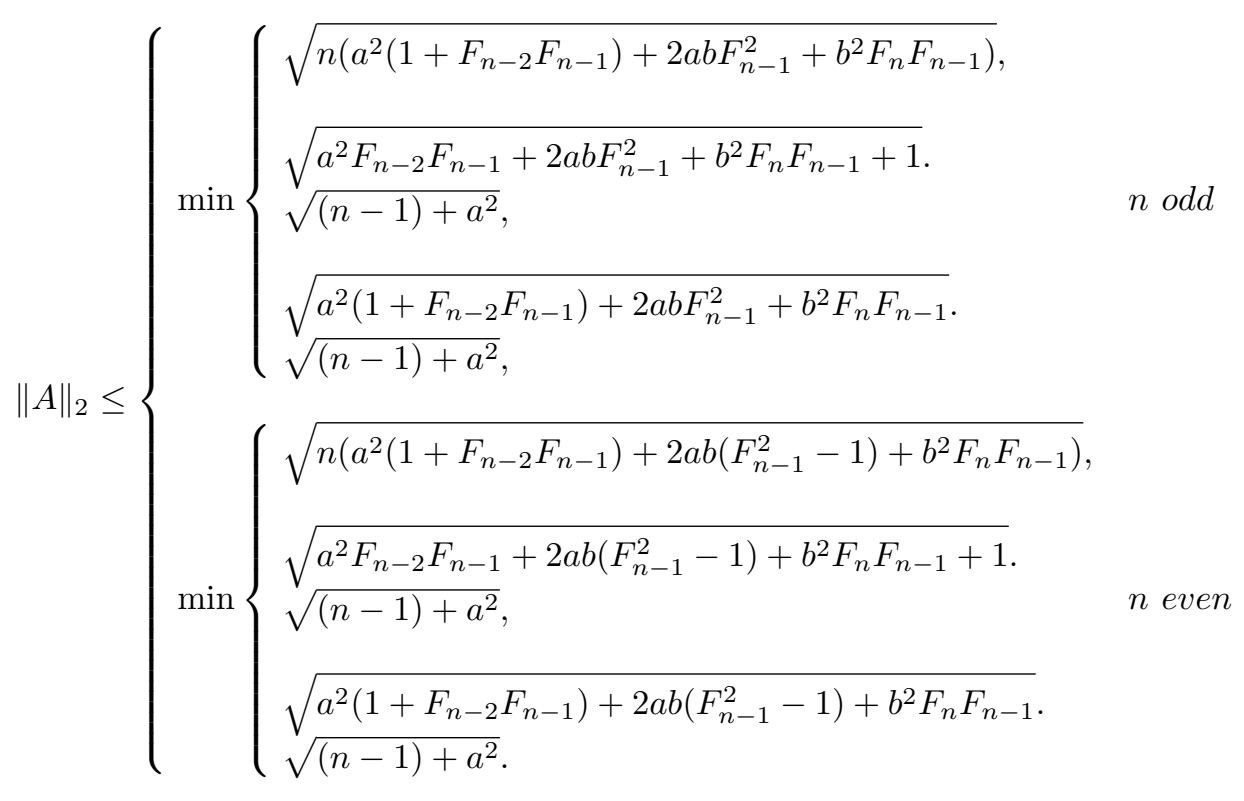

Proof. As

$$
\sum_{k=1}^{n-1} F_{k-1}^{2}=F_{n-1} F_{n-2}, \sum_{k=1}^{n-1} F_{k}^{2}=F_{n} F_{n-1}
$$

and

$$
\sum_{k=1}^{n-1} F_{k-1} F_{k}= \begin{cases}F_{n-1}^{2}, & n \text { odd } \\ F_{n-1}^{2}-1, & n \text { even }\end{cases}
$$

The proof of Theorem 2.6 become trivial.

Corollary 2.7. Let $G_{0}=a=2$ and $G_{1}=b=1$ be in the theorem. As $F_{n} F_{n-1} \geq n-1, \forall n \geq 0$ and as we have $F_{n} F_{n-1}=F_{n-1}^{2}+F_{n-2} F_{n-1}$, then, we obtain

(i) If $|r| \geq 1$, then

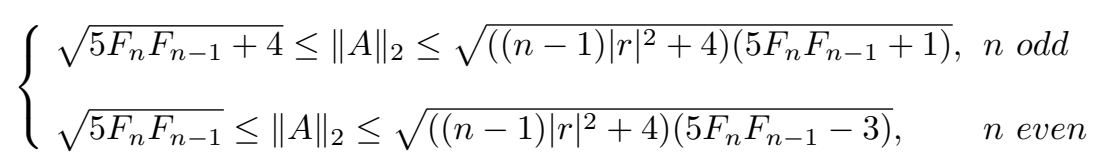

(ii) If $|r|<1$, then

$$
\begin{cases}|r| \sqrt{5 F_{n} F_{n-1}+4} \leq\|A\|_{2} \leq \sqrt{n\left(5 F_{n} F_{n-1}+4\right)}, & n \text { odd } \\ |r| \sqrt{5 F_{n} F_{n-1}} \leq\|A\|_{2} \leq \sqrt{n\left(5 F_{n} F_{n-1}\right)}, & n \text { even }\end{cases}
$$

Acknowledgment: We would like to thank Sä̈d Chandoul and Massöuda Loörayed for helpful discussions and many remarks. 


\section{References}

[1] D. Bozkurt, T. Y. Tam, Determinants and inverses of $r$-circulant matrices associated with a number sequences, Linear and Multilinear Algebra 63(10) (2015) 2079-2088.

[2] H. Civciv, R. Turkmen, Notes on norms of circulant matrices with Lucas numbers, Int. J. Inf. Syst. Sci. 4(1) (2008) 142-147.

[3] C. He, J. Ma, K. Zhang, Z. Wang, The upper bound estimation on the spectral norm of $r$-circulant matrices with the Fibonacci and Lucas numbers, J. Inequal. Appl. Article ID 72 (2015) 1-10.

[4] R. A. Horn, C. R. Johnson, Topics in Matrix Analysis, Cambridge University Press, Cambridge, 1991.

[5] R. Mathias, The spectral norm of nonnegative matrix, Linear Algebra Appl. 139 (1990), 269-284.

[6] A. Nalli, M. Sen, On the norms of circulant matrices with generalized Fibonacci numbers, Selçuk J. Appl. Math. 11(1) (2010) 107-116.

[7] T. Koshy, Fibonacci and Lucas Numbers with Application, John Wiley and Sons, Inc., 2001.

[8] S. Shen, J. Cen, On the norms of $r$-circulant matrices with Fibonacci and Lucas numbers, Appl. Math. Comput. 216(10) (2010) 2891-2897.

[9] S. Solak, On the norms of circulant matrices with Fibonacci and Lucas numbers, Appl. Math. Comput. 160(1) (2005) 125-132.

[10] S. Solak, Erratum to "On the norms of circulant matrices with Fibonacci and Lucas numbers" [Appl. Math. Comput. 160(1) (2005) 125-132], Appl. Math. Comput. 190(2) (2007) 1855-1856.

[11] N. Tuglu, C. Kizilates, On the norms of circulant and $r$-circulant matrices with the hyperharmonic Fibonacci numbers, J. Inequal. Appl. Article ID 253 (2015) 1-11.

[12] S. Vajda, Fibonacci and Lucas numbers and Golden Section, John Wiley and Sons, Inc., 1989. 